\title{
Cooling, Physical Scales and the Vacuum Structure of Y-M Theories.
}

\author{
Margarita García Pérez ${ }^{\mathrm{a}}$, Owe Philipsen ${ }^{\mathrm{b}}$ and Ion-Olimpiu Stamatescu ${ }^{\mathrm{c} *}$ \\ a Departamento de Física Teórica, Univ. Autónoma de Madrid, Canto Blanco, 28049 Madrid, Spain \\ bTheory Division, CERN, CH-1211 Geneva 23, Switzerland \\ ${ }^{\mathrm{c} F E S t, ~ S c h m e i l w e g ~ 5, ~ D-69118 ~ H e i d e l b e r g ~ a n d ~ I T P, ~ P h i l o s o p h e n w e g ~ 16, ~ D-69120 ~ H e i d e l b e r g, ~ G e r m a n y ~}$
}

\begin{abstract}
We present a cooling method controlled by a physical cooling radius that defines a scale below which fluctuations are smoothed out while leaving physics unchanged at all larger scales. This method can be generally used as a gauge invariant low pass filter to extract the physics from noisy MC configurations. Here we apply this method to study topological properties of lattice gauge theories where it allows to retain instanton-anti-instanton pairs.
\end{abstract}

Since MC configurations are noisy at small distances, smoothing is needed to observe the physical structure. One approach is "cooling" [1], a local minimization of a given lattice action. Cooling works as a diffusion process, smoothing out increasingly large regions [2]. Thereby the physical spectrum is affected, in particular the string tension drops rapidly with cooling. Topological excitations may be distorted by bad scaling properties of the action and by instanton - anti-instanton (I-A) annihilation (pairs are not minima of the action). We thus need:

1) to use an action with practically scale invariant instanton solutions and a dislocation threshold to eliminate UV noise (dislocations), and

2) to control cooling by means of a physical scale such that no monitoring or engineering which may introduce uncertainties is necessary.

Restricted Improved Cooling (RIC) fulfills these requirements [3]. RIC preserves physics at scales above a cooling radius $r$ which can be fixed unequivocally beforehand, while smoothing out the structure below $r$. In particular the string tension is preserved beyond $r$, instantons are stable, dislocations are eliminated, and I-A pairs are retained above a threshold defined by $r$.

Properties of RIC. RIC uses the action of the Improved Cooling algorithm (IC) with 5 planar, fundamental Wilson loops [4]. This action is cor-

\footnotetext{
*Partial support from the European Network "Finite Temperature Phase Transitions in Particle Physics".
}

rect to order $\mathcal{O}\left(a^{6}\right)$ and has a dislocation threshold $\rho_{0} \sim 2.3 a$, below which short range topological structure is smoothed out (note that $\rho_{0} \rightarrow 0$ in approaching continuum). Above $\rho_{0}$, instantons are stable to any degree of cooling (however, I-A pairs annihilate). The corresponding improved charge density using the same combination of loops leads to an integer charge already after a few cooling sweeps and stable thereafter.

Here we shall restrict ourselves to $S U(2)$, for the general case see [3]. Recall that the cooling algorithm is derived from the equations of motion

$U_{\mu}(x) W_{\mu}(x)^{\dagger}-W_{\mu}(x) U_{\mu}(x)^{\dagger}=0$,

where $W$ is the sum of staples connected to the link $U_{\mu}(x)$ in the action. It amounts to substitute

$U \rightarrow U^{\prime}=V=\frac{W}{\|W\|},\|W\|^{2}=\frac{1}{2} \operatorname{Tr}\left(W W^{\dagger}\right)$.

We define RIC by the constraint? that only those links be updated, which violate the equation of motion by more than some chosen threshold:

$U \rightarrow V \quad$ iff $\quad \Delta_{\mu}(x)^{2}=\frac{1}{a^{6}} \operatorname{Tr}\left(1-U V^{\dagger}\right) \geq \delta^{2}$.

We have $\Delta_{\mu}^{2}(x) \propto-\operatorname{Tr}\left(\left(D_{\nu} F_{\nu \mu}(x)\right)^{2}\right)$ in continuum limit [5]. Thus $\delta$ controls the energy of the fluctuations around classical solutions and acts as a filter for short wavelengths. Since it uses the same action RIC has the same scaling properties

${ }^{2}$ We thank F. Niedermayer for this suggestion. 
as IC. However, since RIC does not update links already close to a solution, it changes fewer links after every iteration until the algorithm saturates. (3) defines a constrained minimization and the smoothing is homogeneous over the lattice.

The next step is to relate the parameter $\delta$ which defines the cooling to a physical scale. For Yang Mills theory the latter should involve the string tension $\sigma$. We calculate the "effective mass" $M(t)$ from correlation functions of spatial Polyakov loops separated by $t$ steps in time. Asymptotically $M(t) \simeq N_{s} \sigma$ up to finite size corrections.

For the calculations we generate configurations using the Wilson action on the lattices:

$12^{3} \times 36$, p.b.c., $\beta=2.4(a=0.12 \mathrm{fm}): 800$ configurations with 100 sweeps separation;

$24^{4}$, twist in time, $\beta=2.6(a=0.06 \mathrm{fm}): 350$ configurations with 200 sweeps separation.

We do 20000 thermalization sweeps. We use $\delta=$ $2.89,4.92,11.57,23.15$ and $46.30 \mathrm{fm}^{-3}$.

For illustration we present in Fig. $11 M(t)$ for the $24^{4}$ lattice. Defining $r(\delta)$ as the distance $t$ at which $M(t)$ on $\delta$-cooled configurations starts to agree with the uncooled value (obtained by fuzzing and fitted to a smooth function of $t$ ) we arrive at the results in Fig. 2, compatible with

$r(\delta) \simeq 0.8 \delta^{-1 / 3}$,

showing the correct scaling behaviour, as expected since $\delta$ has a continuum limit.

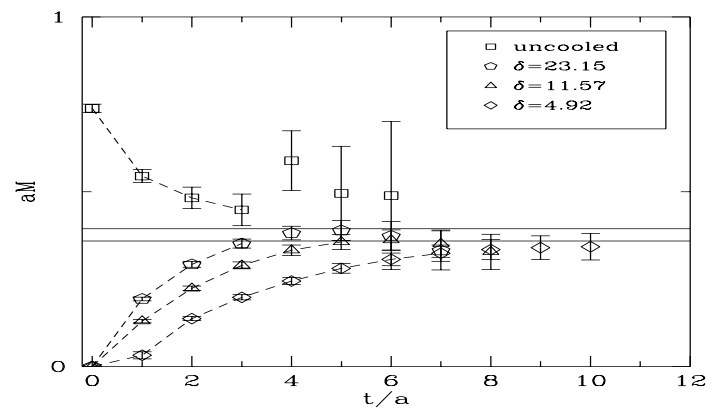

Figure 1. $M(t)$ for the $a=0.12$ fm lattice. The horizontal band represents standard results for $\sigma$.

The behaviour of instantons is similar under RIC and IC whereby the former has a somewhat smaller, $\delta$-dependent dislocation threshold $\rho_{0}(\delta)$. I-A pairs, however, do not annihilate under RIC the way they do for the other cooling methods

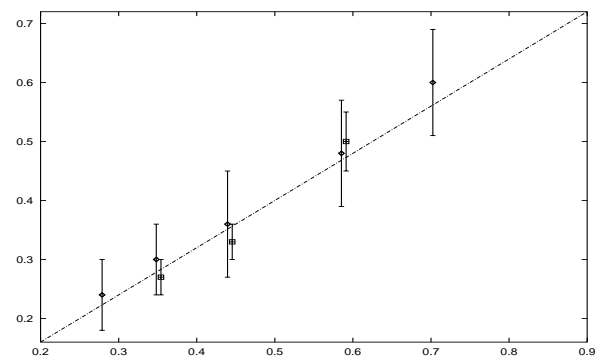

Figure 2. The smoothing scale of RIC: $r(\delta)$ vs $\delta^{-1 / 3}$ for the $a=0.12 \mathrm{fm}$ (diamonds) and $a=0.06 \mathrm{fm}$ (squares) lattices (the middle points are slightly displaced around the true positions for readability). Also shown is the fit (四).

(including IC): since the distortion of the partners in a pair depends on the overlap, RIC preserves pairs below some overlap-threshold. More precisely, it turns out that there is a well defined relation between $\Delta$ and $S_{\mathrm{int}}^{\mathrm{IA}}=16 \pi^{2}-S^{I A}$, therefore RIC stabilizes I-A pairs with $S_{\text {int }}$ below a threshold which is a function of $\delta$, hence of $r$.

SU(2) topological properties by RIC. The total, improved topological charge stabilizes to integer values after only few IC or RIC sweeps. Therefore the topological susceptibility appears very stable if $\delta$ is small enough to eliminate dislocations, shows correct scaling and agrees with the Witten-Veneziano relation.

To describe the details of the instanton ensemble one needs to recognize the particular I's and A's. Since the objects can be very distorted, our description relies on two assumptions: a) instantons should appear as local self-dual peaks in the action and charge density, and b) only pairs with $S_{\text {int }}^{\text {IA }}$ considerably smaller than $16 \pi^{2}$ should be considered as such. We approximate the action and charge density by a superposition of I's and A's parameterized through the BPST formula for an instanton of size $\rho$. We measure the adequacy of this ansatz through the fraction of the total action (charge) reproduced in this way. We represent the data in physical units using the corresponding $a$ and the $r(\delta)$ obtained from the string tension analysis without any further fit or tuning.

The main results of our analysis are:

1. The density of instantons increases drastically with decreasing smoothing scale $r$. 


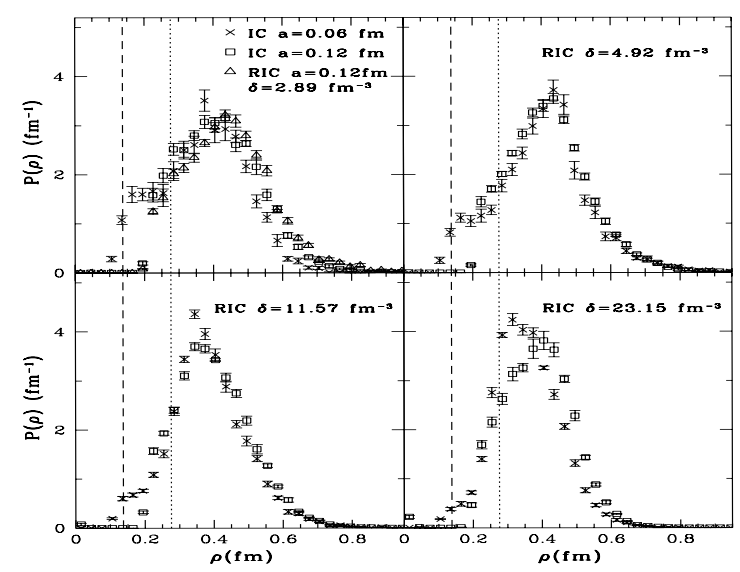

Figure 3. Normalized size distributions on the $a=0.12 \mathrm{fm}$ lattice (squares, triangles) and $a=$ $0.06 \mathrm{fm}$ lattice (crosses). Vertical dotted (dashed) lines indicate the $I C$ dislocation threshold $\rho_{0}=$ $2.3 a$ for $a=0.12$ (0.06) $\mathrm{fm}$.

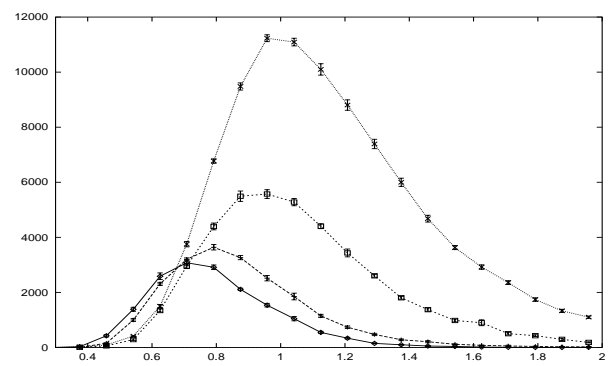

Figure 4. Non-normalized overlap distributions for $a=0.06 \mathrm{fm}$ and $\delta=2.89$ (circles), 4.92 (bars), 11.57 (squares) and 23.15 (crosses) $\mathrm{fm}^{-3}$.

2. Size distributions depend only weakly on $r$.

3. The typical I-A distance $d_{I A}$ (from an I to the nearest A) seems given by the smoothing scale.

4. The overlap $\left(\rho_{I}+\rho_{A}\right) / 2 d_{I A}$ increases strongly with decreasing $r$ and the fit quality deteriorates. All these results are largely invariant under rescaling of the lattice spacing $a$. They are summarized in the Figures 3 , 4 and 5 .

It appears therefore that there are topological features of the vacuum structure, like susceptibility and charge distributions and to a fair degree also size distributions, which are well defined, show correct scaling and are rather independent on the smoothing scale once the noise has been damped sufficiently. However, I(A)-density, overlap and I-A distance distributions are not of this kind: although these quantities seem to scale cor-

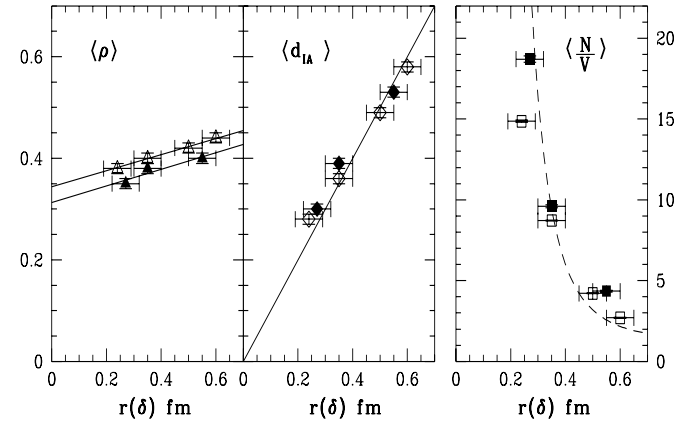

Figure 5. Dependence of the average size (triangles), the average $I$ - A distance (diamonds) and the instanton density (squares) on the cooling radius $r$. The dashed curve is $\langle N / V\rangle=$ $\langle N / V\rangle_{\text {asymptotic }}+C / r^{4}$. Open (filled) symbols correspond to the $a=0.12$ (0.06) fm lattice.

rectly with the cut off, their behaviour with $r$ suggests that looking at smaller and smaller scales a continuous spectrum of fluctuations emerges and a description in terms of I's, A's and I-A pairs becomes less and less meaningful. This may explain both the agreements and the disagreements between various analyses - see, e.g. [6].

Generally RIC proves itself a good instrument for probing different scales in a controlled way and extract physics from noisy configurations.

\section{REFERENCES}

1. B. Berg, Phys. Lett. B104 (1981) 475; M. Teper, Phys. Lett. B162 (1985) 357.

2. M. Campostrini, A. Di Giacomo, M. Maggiore, H. Panagopoulos and E. Vicari, Phys. Lett. B225 (1989) 403.

3. M. García Pérez, O. Philipsen and I.-O. Stamatescu, Nucl. Phys. B551 (1999) 293.

4. P. de Forcrand, M. García Pérez and I.O. Stamatescu, Nucl. Phys. Proc. Suppl. 47 (1996) 777; Nucl. Phys. B499 (1997) 409.

5. M. García Pérez and P. van Baal, Nucl. Phys. B429 (1994) 451.

6. T. DeGrand, A. Hasenfratz and D. Zhu, Nucl. Phys. B475 (1996) 321; M. Feurstein, E. M. Ilgenfritz, M. Müller-Preussker and S. Thurner, Nucl. Phys. B511 (1998) 421; T. De Grand, A. Hasenfratz and T. G. Kovacs, Nucl. Phys. B520 (1998) 301; D. A. Smith and M. Teper, Phys. Rev. D58 (1998) 014505. 